PROCEEDINGS OF THE

AMERICAN MATHEMATICAL SOCIETY

Volume 129, Number 12, Pages 3737-3740

S 0002-9939(01)06358-4

Article electronically published on July 10, 2001

\title{
NEW PROOF OF TWO BERSTEIN-HILTON THEOREMS
}

\author{
CARMEN ELVIRA AND JOSE L. NAVARRO \\ (Communicated by Ralph L. Cohen)
}

\begin{abstract}
With arguments of Homotopy Theory and without the assumption of finite type, we give short proofs of two classic theorems which deal with the co- $H$-type of a suspension and the homotopy class of a suspension map.
\end{abstract}

\section{INTRODUCTION}

In this paper we work in the based category of spaces having the homotopy type of $C W$-complexes with compactly generated topologies, so $\operatorname{dim} X \leq n$ means that there is an $n$-dimensional $C W$-complex in the homotopy type of $X$. The flat product $A b B$ is the homotopy fibre of the inclusion map $j: A \vee B \longrightarrow A \times B$. Clearly, $\zeta=M\left(\Omega i_{1} \times \Omega i_{2}\right)$ is an explicit coretraction for $\Omega j$, where $M$ denotes the loop multiplication on $\Omega(A \vee B)$. In the sequel we shall use the same symbol for a map and its homotopy class, thus we mark as equal homotopic maps. The class $[*]$ of the nullhomotopic maps will be denoted by 0 .

Since $(\Omega j)(1-\zeta(\Omega j))=0$, there is a map $\xi: \Omega(A \vee B) \longrightarrow \Omega(A b B)$, unique up to homotopy, such that $(\Omega \pi) \xi=1-\zeta(\Omega j)$ and the map $J(f): \Omega X \longrightarrow \Omega(A b B)$ given by the composition $J(f)=\xi(\Omega f)$ is called the co-Hopf construction associated to the map $f: X \longrightarrow A \vee B$.

A comultiplication on a space $X$ is a map $\mu: X \longrightarrow X \vee X$ such that $j \mu=\Delta$, and we say that $\mu$ is homotopy associative if $(\mu \vee 1) \mu=(1 \vee \mu) \mu$. We denote by $\sigma$ the standard comultiplication on any suspension space. A map $f:(X, \mu) \longrightarrow(Y, \nu)$ is a co- $H$-map, with respect to $\mu$ and $\nu$, if $\nu f=(f \vee f) \mu$ or, equivalently, if $\left(i_{1}+i_{2}\right) f=i_{1} f+i_{2} f$ in $[X, Y \vee Y]$. Plainly, the suspension $\Sigma g$ of any map $g: A \longrightarrow B$ is a co- $H$-map, with respect to the standard comultiplications.

With arguments of Homology Theory, and restricted to $C W$-complexes of finite type (i.e. $C W$-complexes with finitely generated homology groups), Berstein and Hilton proved in [1] the two following results:

Theorem A. Let $(X, \mu)$ be an $(n-1)$-connected co-H-space, $n \geq 2$, such that $\operatorname{dim} X \leq 3 n-3$. Then $X$ has the co-H-type of a suspension.

Theorem B. Let $B$ be an $(n-1)$-connected space, $n \geq 1$, and let $A$ be such that $\operatorname{dim} A \leq 3 n-2$. Then every co-H-map $f: \Sigma A \longrightarrow \Sigma B$ is homotopic to a suspension map.

Received by the editors March 20, 2000.

2000 Mathematics Subject Classification. Primary 55P40, 55P45; Secondary 55P30.

Key words and phrases. Co- $H$-space, suspension, coprojective plane.

The first author was partially supported by DGS, project PD96-0740. 
In the introduction of [1] the authors suggest that a simpler proof of their results should be possible. In this paper we give short proofs of these theorems in the same spirit as the original proofs, but with arguments of Homotopy Theory that make unnecessary the assumption of finite type.

\section{The Coprojective Plane of a CO- $H$-SPACE}

Let $(X, \mu)$ be a co- $H$-space. In 4 Hilton defined the coprojective plane $X C_{2}$ of $X$ as the homotopy fibre of the co-Hopf construction $J(\mu)$. Let $f:(X, \mu) \longrightarrow(Y, \nu)$ be a co- $H$-map. Since the co-Hopf construction is natural, the right square $D$ in the following diagram is homotopy commutative:

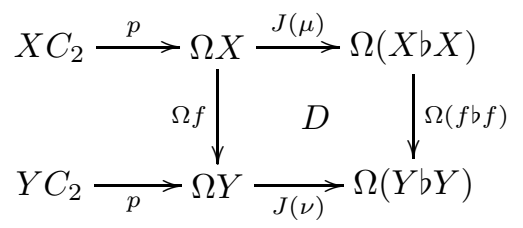

Therefore $J(\nu)(\Omega f) p=0$ or, equivalently, there is a map $f_{2}: X C_{2} \longrightarrow Y C_{2}$ such that $p f_{2}=(\Omega f) p$. In particular, the coprojective plane is an invariant of the co- $H$-type in the 1 -connected $C W$-type.

Composition of $(\Omega \pi) \xi=1-\zeta(\Omega j)$ with $\Omega \mu$ yields $(\Omega \pi) J(\mu)=\Omega \mu-\zeta(\Omega j)(\Omega \mu)=$ $\Omega \mu-\zeta(\Omega \Delta)=\Omega\left(i_{1}+i_{2}\right)-\left(\Omega i_{1}+\Omega i_{2}\right)$. Let $f:(\Sigma A, \sigma) \longrightarrow(X, \mu)$ be a map. Then

$$
\begin{aligned}
(\Omega \pi) J(\mu)(\Omega f) e & =\Omega\left(\left(i_{1}+i_{2}\right) f\right) e-\left(\Omega\left(i_{1} f\right) e+\Omega\left(i_{2} f\right) e\right) \\
& =\mathcal{A}\left(\left(i_{1}+i_{2}\right) f-\left(i_{1} f+i_{2} f\right)\right),
\end{aligned}
$$

where $\mathcal{A}$ is the adjointness isomorphism and $e: A \longrightarrow \Omega \Sigma A$ is the unit. Since $(\Omega \pi)_{*}$ is a monomorphism, one has the following result which appears in [4] without proof.

Proposition 2.1. A map $f: \Sigma A \longrightarrow X$ is a co-H-map, with respect to $\sigma$ and $\mu$, if and only if $J(\mu) \mathcal{A}(f)=0$.

In other words, $f$ is a co- $H$-map if and only if its adjoint $\mathcal{A}(f)$ factors through $X C_{2}$. Obviously, $p: X C_{2} \longrightarrow \Omega X$ factors through $X C_{2}$ and, since $\mathcal{A}^{-1}(p)=\varepsilon(\Sigma p)$, one has

Corollary 2.2. The composition $\varepsilon(\Sigma p)$ is a co-H-map.

There is a triple $\Omega X \stackrel{J(\mu)}{\longrightarrow} \Omega(X b X) \stackrel{q}{\longrightarrow} X$, where $q=0$, which is a wordby-word dual of the Sugawara fibration. However, Hilton proved that this triple is not a cofibration. Consider the commutative diagram

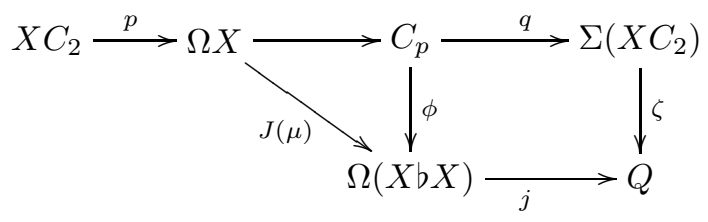

where $Q$ is the homotopy cofibre of $J(\mu)$. If $X$ is $(n-1)$-connected, $n \geq 2$, then $\Omega(X b X)$ is $(2 n-3)$-connected, and $J(\mu)$ is an $(n-1)$-equivalence. By a result of Ganea ([2], Theorem 1.1), the extension $\phi$ of $J(\mu)$ is a $(3 n-3)$-equivalence and 
likewise for $\zeta$, by the standard 5-lemma argument. Let $k: Q \longrightarrow \Sigma \Omega X$ be the next map in the Puppe sequence of $J(\mu)$.

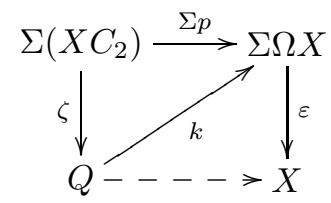

Then $k \zeta=\Sigma p$ and, if $h: Q \longrightarrow X$ is defined by composition $h=\varepsilon k$, Theorem 3.4 in [5] implies that $h$ is a $(3 n-3)$-equivalence. Since $h \zeta=\varepsilon k \zeta=\varepsilon(\Sigma p)$, we then have the following.

Proposition 2.3. Let $X$ be an $(n-1)$-connected co-H-space, $n \geq 2$. Then the composition $\varepsilon(\Sigma p)$ is a $(3 n-3)$-equivalence.

\section{Proof of Theorems A And B}

Let $X$ be a 0-connected $C W$-complex and let $\phi: \widetilde{H}_{n}(X) \longrightarrow F$ be an epimorphism of $\widetilde{H}_{n}(X)$ into the free abelian group $F$, where $n \geq 2$. Then there is a subcomplex $X(n, F)$ of $X$ with the same $(n-1)$-skeleton of $X$, and such that

(1) $u_{*}: \widetilde{H}_{q}(X(n, F)) \longrightarrow \widetilde{H}_{q}(X)$ is an isomorphism for every $q<n$,

(2) $\phi u_{*}: \widetilde{H}_{n}(X(n, F)) \longrightarrow F$ is an isomorphism,

(3) $\widetilde{H}_{q}(X(n, F))=0$ for every $q>n$,

where $u: X(n, F) \longrightarrow X$ denotes the inclusion map.

Note that, if $F=0$ is the trivial group, then $X(n, 0)=X(n-1)$ is the homology $(n-1)$-section of $X$. This (modified) homology decomposition was given in [1].

Assume that $(X, \mu)$ is an $(n-1)$-connected co- $H$-space, $n \geq 2$, such that $\operatorname{dim} X \leq$ $3 n-3$, and let $Y$ be a $C W$-complex in the homotopy type of $X C_{2}$. If we denote $W=$ $Y(3 n-4, F)$ and $u: W \longrightarrow Y$ is the inclusion map, $F=\widetilde{H}_{3 n-3}(X)$ being a free abelian group, then Corollary 2.2 and Proposition 2.3 imply that the composition

$$
\Sigma W \stackrel{\Sigma u}{\longrightarrow} \Sigma Y \simeq \Sigma\left(X C_{2}\right) \stackrel{\Sigma p}{\longrightarrow} \Sigma \Omega X \stackrel{\varepsilon}{\longrightarrow} X
$$

is a co- $H$-equivalence and Theorem $\mathrm{A}$ is proved.

Now, let $f: \Sigma A \longrightarrow \Sigma B$ be a co- $H$-map with respect to $\sigma_{A}$ and $\sigma_{B}$. Then it follows that $J\left(\sigma_{B}\right)(\Omega f) e_{A}=0$, and so there is a map $u: A \longrightarrow(\Sigma B) C_{2}$ such that $p u=(\Omega f) e_{A}$.

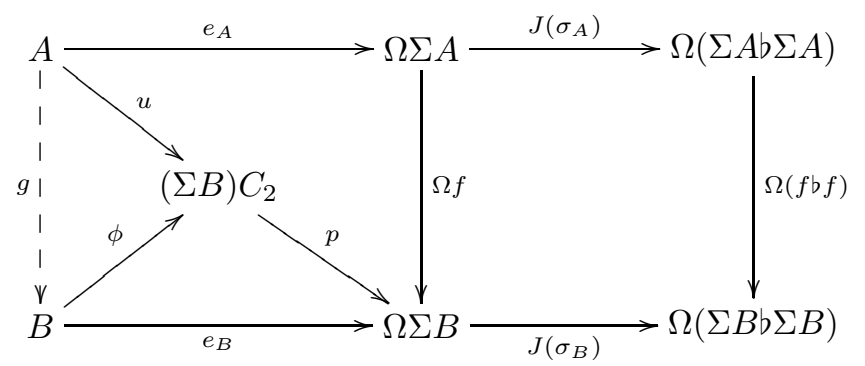

On the other hand, since $1: \Sigma B \longrightarrow \Sigma B$ is obviously a co- $H$-map, Proposition 2.1 implies that $J\left(\sigma_{B}\right) e_{B}=0$. Then there is a map $\phi: B \longrightarrow(\Sigma B) C_{2}$ such that $p \phi=e_{B}$. Assume that $B$ is $(n-1)$-connected; then $\Sigma B$ is $n$-connected and, by 
applying Proposition 2.3 to $\Sigma B$, we infer that $\varepsilon(\Sigma p)$ is a $3 n$-equivalence. Note that $\varepsilon(\Sigma p)(\Sigma \phi)=\varepsilon\left(\Sigma e_{B}\right)=1$, so $\Sigma \phi$ is a coretraction of $\varepsilon(\Sigma p)$. Thus, $\Sigma \phi$ is a $(3 n-1)$-equivalence, and so $\phi$ is a $(3 n-2)$-equivalence. Hence, the induced map $\phi_{*}:[A, B] \longrightarrow\left[A,(\Sigma B) C_{2}\right]$ is bijective if $\operatorname{dim} A<3 n-2$, and surjective if $\operatorname{dim} A=3 n-2$. Therefore, provided $\operatorname{dim} A \leq 3 n-2$, there is a map $g: A \longrightarrow B$ such that $\phi g=u$. Thus, $e_{B} g=p \phi g=p u=(\Omega f) e_{A}=\mathcal{A}(f)$ and, by taking adjoints, we have $f=\mathcal{A}^{-1}\left(e_{B} g\right)=\Sigma g$. This proves Theorem B.

\section{REMARK}

It has been known for a long time that any $(n-1)$-connected space such that $\operatorname{dim} X \leq 2 n-1, n \geq 1$, has the homotopy type of a suspension. A proof, by induction on the geometrical dimension of a $C W$-complex, was given in [3]. After the modified homology decomposition of Berstein-Hilton it is possible to give a shorter proof. Indeed, if $n \geq 2$ the hypotheses imply that $\varepsilon: \Sigma \Omega X \longrightarrow X$ is a $(2 n-1)$-equivalence, and that $F=\widetilde{H}_{2 n-1}(X)$ is a free abelian group, respectively. Let $Y$ be a $C W$-complex in the homotopy type of $\Omega X$. If $W=Y(2 n-2, F)$ and $u: W \longrightarrow Y$ is the inclusion map, then the composition

$$
\Sigma W \stackrel{\Sigma u}{\longrightarrow} \Sigma Y \simeq \Sigma \Omega X \stackrel{\varepsilon}{\longrightarrow} X
$$

is a homotopy equivalence in the 1 -connected $C W$-type. If $n=1$, note that $\operatorname{dim} X \leq$ 1 implies that $X$ is contractible or $X$ has the homotopy type of a wedge of 1 -spheres, so the suspension of a discrete space.

\section{REFERENCES}

1. I. Berstein and P. Hilton, On suspensions and comultiplications, Topology 2 (1963), 73-82 MR 27:762

2. T. Ganea, A generalization of the homology and homotopy suspension, Comment. Math. Helv. 39 (1965), 295-322 MR 31:4033

3. P. Hilton, On divisors and multiples of continuous maps, Fundamenta Math. 43 (1956), 358386 MR 18:814b

4. P. Hilton, Note on a theorem of Stasheff, Bull. Polish Acad. Sci. Math. 10 (1962), 127-131 MR 25:4536

5. M. Walker, Homotopy pullbacks and applications to duality, Canad. J. Math. 29 (1977), 45-64 MR 55:11245

Departamento de Analisis Economico, Universidad de Zaragoza, 50005-Zaragoza, SPAIN

E-mail address: celvira@posta.unizar.es

Departamento de Matematicas, Universidad de Zaragoza, 50009-Zaragoza, Spain

E-mail address: jlnava@posta.unizar.es 